\title{
microRNA-30b/c inhibits non-small cell lung cancer cell proliferation by targeting Rab18
}

\author{
Keng Zhong ${ }^{1+}$, Kun Chen ${ }^{2+}$, Lin Han ${ }^{1 *}$ and Bailing $\mathrm{Li}^{1 *}$
}

\begin{abstract}
Background: MicroRNAs (miRNAs) are small noncoding RNAs that post-transcriptional regulate gene expression in a variety of cancers. Increasing evidences indicate that miR-30 expression is down-regulated in numerous human cancers including non-small cell lung cancer (NSCLC) which hypothesizes that miR-30 may play an important role in tumorigenesis. The aim of this study was to investigate the target gene of miR-30 and its roles in tumor growth of NSCLC.

Methods: Luciferase reporter assays were employed to validate regulation of a putative target of miR-30. The effect of miR-30 on endogenous levels of this target were subsequently confirmed via Western blot (WB). Quantitative reverse transcription-polymerase chain reaction (qRT-PCR) was performed to determine the expression level of miR-30 in NSCLC specimens and adjacent non-tumor tissues. MTT assays were conducted to explore the impact of miR-30 overexpression on the proliferation of human NSCLC cells.

Results: Both miR-30b and miR-30c (miR-30b/c) were found having target site in same region of Rab18 mRNA. Luciferase assays using a reporter carrying a putative miR-30b/c target site in the coding DNA sequence (CDS) region of Rab18 revealed that miR-30b/c directly targeted Rab18. Overexpression of miR-30b/c led to down-regulation of Rab18 in A549 and H23 cells at protein levels but not mRNA levels. Down-regulation of miR-30b/c and up-regulation of Rab18 protein levels were detected in NSCLC specimens compared with adjacent non-tumor tissues. Overexpression of miR-30b/c suppressed NSCLC cells growth. Knockdown of Rab18 by siRNA significantly inhibited the proliferation of NSCLC cells.
\end{abstract}

Conclusions: We demonstrated that miR-30b/c was down-regulated in NSCLC specimens compared with adjacent non-tumor tissues. miR-30b/c directly targeted and down-regulated Rab18 expression and inhibited NSCLC cells proliferation. These data indicated that miR-30b/c could serve as a tumor suppressor gene involved in NSCLC pathogenesis.

Keywords: miR-30b, miR-30c, Proliferation, Rab18, NSCLC

\section{Background}

Lung cancer is the most common cause of cancer-associated deaths worldwide, especially for male [1]. NSCLC accounts for nearly $80 \%$ of lung cancers [2]. NSCLC population has grown quickly over the past five years in China [3]. Although surveillance and clinical treatment strategies have been improved, the 5-year survival of NSCLC patients after curative resection is reported to be only 30-60\% [4]. Therefore, elucidating the potential mechanism

\footnotetext{
*Correspondence: sh_hanlin@163.com; smmu_libailing@163.com

'Equal contributors

'Department of Cardiothoracic Surgery, Changhai Hospital affiliated to Second Military Medical University, Shanghai 200433, China

Full list of author information is available at the end of the article
}

that mediate the initiation and progression of NSCLC is urgent and of great interest.

miRNAs are a class of small non-coding RNAs which plays an important role in post-transcriptional regulation in various biological processes. Mechanistically, miRNAs bind to their target mRNAs and cause translation to be blocked or mRNA degradation [5,6]. Accumulating evidences have suggested that miRNAs play diverse roles in tumorigenesis and cancer progression [7-10]. In recent years, miRNAs have received great attention in NSCLC research. Several deregulated miRNAs in NSCLCs such as miR-221, miR-222, miR-449a, miR-21, miR-205, miR-10b, miR-143 and miR-181a have been shown to regulate cell 
growth, apoptosis, migration and invasion [11-16]. These findings indicate that deregulation of miRNA expression may be associated with tumorigenesis of NSCLCs.

miR-30 is significantly down-regulated in several cancers, including breast cancer [17], malignant peripheral nerve sheath tumors [18], glioma [19], and lung cancer [20]. As the down-regulation of miR-30 is related to a number of cancers, it has been hypothesized that miR-30 may play an important role in tumorigenesis and tumor development. However, the function of miR-30 especially in NSCLC remains unclear.

In our study, we showed that Rab18 were identified as direct functional targets of miR-30b/c in NSCLC cells and $\mathrm{miR}-30 \mathrm{~b} / \mathrm{c}$ was down-regulated in NSCLC tissues compared to adjacent non-tumor tissues. Furthermore, ectopic overexpression of miR-30b/c blocked tumor cell proliferation in vitro. These data suggested that the reduced expression of $\mathrm{miR}-30 \mathrm{~b} / \mathrm{c}$ might facilitate the development of NSCLCs.

\section{Methods \\ Specimens}

In this study, 5 paired NSCLC and adjacent non-tumor specimens were collected from the Department of Respiratory Medicine, the Second Affiliated Hospital, Second Military Medical University (Shanghai, China). All tissue samples were flash-frozen in liquid nitrogen immediately after collection and stored at $-80^{\circ} \mathrm{C}$ until use. The study protocol was approved by Shanghai Changzheng Hospital Ethical Committee. Informed consent was obtained from all patients. All clinic pathologic and biological data were available for those patients. Both tumor and non-tumor samples were confirmed by pathological examination. No patients received chemotherapy or radiotherapy prior to surgery.

\section{Cell culture}

The human NSCLC cell lines A549 and H23 were purchased from ATCC. A549 cells were cultured in Ham's F12K media (Invitrogen, Carlsbad, CA, USA) supplemented with $10 \%$ (vol/vol) fetal bovine serum (FBS) (Invitrogen, Carlsbad, CA, USA), H23 cells were cultured in Dulbecco's Modified Eagle Medium (DMEM; SigmaAldrich, St. Louis, Mo., USA) supplemented with 10\% (vol/vol) FBS, HEK293 cells were purchased from ATCC and grown were cultured in DMEM media containing $10 \%$ (vol/vol) FBS. Cells were maintained at $37^{\circ} \mathrm{C}$ in a humidified atmosphere with $5 \% \mathrm{CO}_{2}$.

\section{RNA isolation and qRT-PCR}

Total RNA was isolated from NSCLC tissues, adjacent non-tumor tissues and cell lines using Trizol according to the manufacturer's instructions. qRT-PCR detection was performed as described previously [21]. U6 small
RNA was used as an internal control for normalization and quantification of $\mathrm{miR}-30 \mathrm{~b} / \mathrm{c}$ expression. $\beta$-actin was used as an internal control for normalization and quantification of Rab18 expression. All primers were listed in Table 1.

\section{Western blot}

The Western blot protocol was described previously [22]. Proteins were separated on a $12 \%$ SDS-PAGE gel and transferred onto a nitrocellulose membrane (Bio-Rad, Hercules, USA). The membrane was blocked with $5 \%$ non-fat milk and incubated with anti-Rab18 antibody (Proteintech Group) or anti-beta-actin antibody (Sigma, CA, USA). After being washed extensively, secondary antibody (Pierce, IL, USA) was added to the system. Immunoreactive protein bands were detected using an Odyssey Scanning system.

\section{Oligonucleotides transfection}

RNA oligonucleotides were chemically synthesized and purified by Genepharma Co. Ltd., (Shanghai, China). Sequence of human miR-30b mimics was $5^{\prime}$ - UGU AAA CAUC CUA CAC UCA GCU -3' and human miR-30c mimics was 5' - UGU AAA CAU CCU ACA CUC UCA GC $-3^{\prime}$. Negative control oligonucleotides for miRNA mimics was $5^{\prime}$-CAG UAC UUU UGU GUA GUA CAA$3^{\prime}$. The sequences of Rab18 siRNA was: 5' - GAA ACA UAC UGU ACA AGA ATT -3' (sense) and 5'-UUC UUG UAC AGU AUG UUU CTT-3' (antisense), Control siRNA was: 5'-UUC UCC GAA CGU GUC ACG

\section{Table 1 All primers used in this study}

\begin{tabular}{|c|c|}
\hline Name & Primer sequence \\
\hline Rab18 (WT) F & 5'-AAACTAGTTAACTCCCAGCTATTATAG-3' \\
\hline Rab18 (WT) R & 5'-GGAAGCTTTCTTCTTGTGACATCATAAAC-3' \\
\hline Rab18 (MUT) F & 5'-AAACTAGTTAACTCCCAGCTATTATAG-3' \\
\hline Rab18 (MUT) R & 5'-GGAAGCTTTCTTCTTCAGTGTAGAATATGTAA-3' \\
\hline U6 RT & $\begin{array}{l}\text { 5'-GTCGTATCCAGTGCAGGGTCCGAGGTATTCGCAC } \\
\text { TGGATACGACTGGAAC-3' }\end{array}$ \\
\hline U6 F & 5'-AACGCTTCACGAATTTGCGT-3' \\
\hline miR-30b RT & $\begin{array}{l}\text { 5'-GTCGTATCCAGTGCAGGGTCCGAGGTATTCGCAC } \\
\text { TGGATACGACAGCTGA-3' }\end{array}$ \\
\hline miR-30b F & 5'-ATCGCTGTAAACATCCTACAC-3' \\
\hline miR-30c RT & $\begin{array}{l}\text { 5'-GTCGTATCCAGTGCAGGGTCCGAGGTATTCGCAC } \\
\text { TGGATACGACGCTGAG-3' }\end{array}$ \\
\hline miR-30c F & 5'-GCTTTGTAAACATCCTACACT-3' \\
\hline Rab18 F & 5'-TAAAGAGCCAGATAGGAA-3' \\
\hline Rab18 R & 5'-TCTATAATAGCTGGGAGTT-3' \\
\hline$\beta$-actin F & 5'-AGCAGCATCGCCCCAAAGTT-3' \\
\hline$\beta$-actin R & 5'-GGGCACGAAGGC TCATCATT-3' \\
\hline miRNA Universal R & 5'-GTGCAGGGTCCGAGGT-3' \\
\hline
\end{tabular}

Abbreviations: $R T$ reverse-transcription primer, $F$ forward primer, $R$ reverse primer, WT wild type, MUT mutant. 
UTT-3' (sense), 5' -ACG UGA CAC GUU CGU AGA ATT-3' (antisense). The transfections were performed with INTERFERin reagent (Polyplus-transfection). The final concentration of miRNA was $50 \mathrm{nM}$. The final concentration of siRNA was $20 \mathrm{nM}$.

\section{Luciferase assay}

Luciferase reporter construct was made by cloning human Rab18 sequence containing the potential miR-30b/c binding site into pMIR-Report construct (Ambion, Austin, USA). Wild type (WT) or mutant (MUT) Rab18 fragment (from 501 to 562) was amplified by PCR using the primers in Table 1. All fragments were amplified and cloned into the luciferase repoter via SpeI and HindIII sites. Luciferase reporter assays were performed as previously [22], HEK293 cells were co-transfected with $50 \mathrm{nM}$ miRNA mimics or negative control oligonucleotides, $100 \mathrm{ng}$ of firefly luciferase reporter and $20 \mathrm{ng}$ of pRL-TK (Promega, USA) using the JetPRIME reagent (Polyplus-transfection). Cells were collected 48 hours after last transfection and analyzed using Dual-Luciferase Reporter Assay System (Promega).

\section{MTT assay}

The in vitro growth of NSCLC cells was measured using the MTT assay. 5000 cells were seeded into each well of 96-well plates and transfected with miRNA mimics or negative control oligonucleotides at a final concentration of $50 \mathrm{nM}$ respectively. On the day of harvest, $100 \mu \mathrm{l}$ of

A

501-562 Rab18 mRNA (WT):

5'-UAACUCCCAGCUAUUAUAGAGGUGCACAGGGUGUUAUAUUAGUUUAUGAUGUC-ACAAGAAGA-3'

III | | | || || || |

miR-30b: 3'-CGACUCUCACAUCCUACAAAUGU-5'

miR-30c: 3'-UCGACUCACAUCCUACAAAUGU-3'

501-562 Rab18 mRNA (MUT):

I

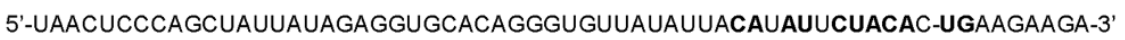

B

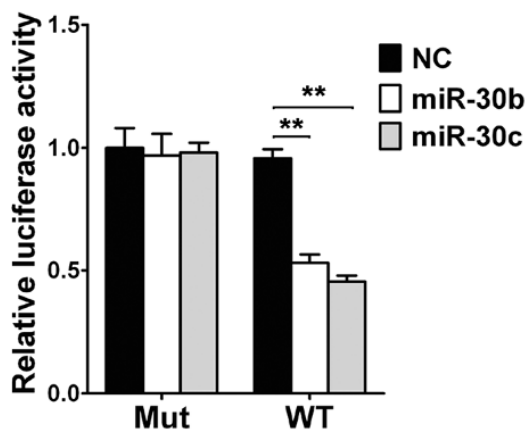

D

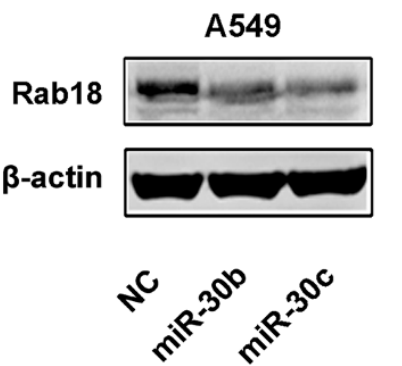

C

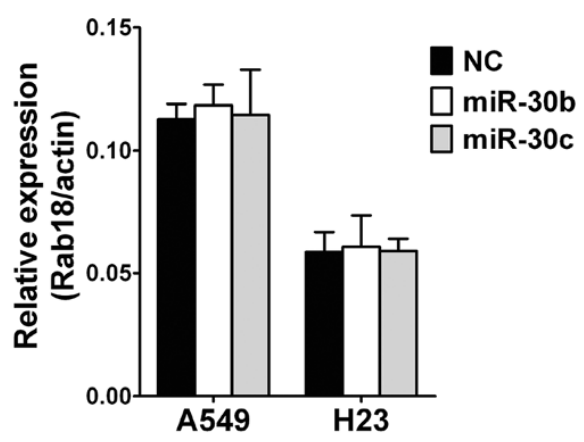

H23

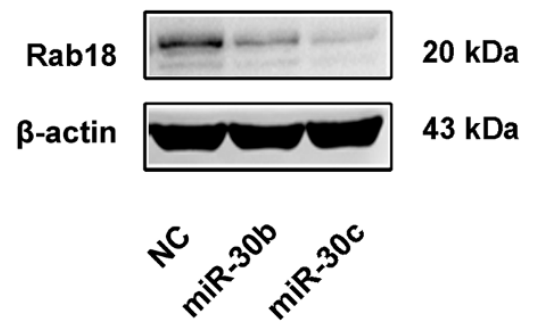

Figure 1 miR-30b/c targets Rab18. (A) Wild-type (WT) and mutant (Mut) of putative miR-30b and miR-30c targeting sequences in Rab18 mRNA. Mutant sequences were shown in bold type. (B) Analysis of luciferase activity. HEK293 cells were cotransfected with miR-30b or miR-30c mimics and negative control oligonucleotides, pRL-TK and firefly luciferase reporter plasmid containing putative miR-30b/c targeting sequences of Rab18. pRL-TK was cotransfected as an internal control to correct the differences in both transfection and harvest efficiencies. The firefly luciferase activity of each sample was normalized to the Renilla luciferase activity. The normalized luciferase activity control oligo nucleotides was set as relative luciferase activity 1 respectively. (C,D) Effects of miR-30b/c on the endogenous Rab18 expression levels. A549 and H23 cells were cotransfected with miR-30b or miR-30c mimics and negative control oligonucleotides. Forty-eight hours after transfection, cells were isolated, the mRNA levels (C) and protein levels (D) of Rab18 were analyzed by qRT-PCR and WB, respectively. ( ${ }^{*} P<0.05,{ }^{* *} P<0.01$, Student's $t$-test). 
spent medium was replaced with an equal volume of fresh medium containing MTT $0.5 \mathrm{mg} / \mathrm{ml}$. Plates were incubated at $37^{\circ} \mathrm{C}$ for $4 \mathrm{hrs}$, then the medium was replaced by $100 \mu \mathrm{l}$ of DMSO (Sigma) and plates shaken at room temperature for $10 \mathrm{~min}$. The absorbance was measured at $570 \mathrm{~nm}$.

\section{Immunohistochemistry}

Immunohistochemical (IHC) staining of samples was performed as previously reported [23]. Paraffin-embedded tissue sections were deparaffinized in xylene and rehydrated in graded series of ethanol followed by heat induced epitope retrieval in citrate buffer (PH 6.0). Rab18 expression were detected using anti-Rab18 polyclonal antibody (Proteintech Group).

\section{Statistical analysis}

Data are presented as the mean \pm standard deviation from at least three independent experiments. The two-tailed $t$-test was used to draw a comparison between groups. The null hypothesis was rejected at the 0.05 level.

\section{Results}

miR-30b/c directly targets Rab18 in human NSCLC cells

In order to investigate the biological significance and its underlying mechanisms of the silenced miR-30 in NSCLC. As miRNAs are a group of post-transcriptional gene regulators which potentially play a critical role in tumorigenesis by regulating the expression of their target genes, the target genes of miR-30 that functioned in NSCLC pathogenesis was further analyzed. Newly published CLASH data in HEK293 cells provided us with the direct evidence for miRNA:mRNA pairing [24]. The CLASH data showed that both miR-30b and miR-30c targeted in coding DNA sequence of Rab18 which was associated with proliferation in hepatocellular carcinoma [23]. To confirm whether $\mathrm{miR}-30 \mathrm{~b} / \mathrm{c}$ regulated the expression of Rab18 gene, we first performed luciferase reporter assays in HEK293 cells. Our results showed that the reporter plasmid with wildtype targeting sequence of Rab18 mRNA caused a significant decrease in luciferase activity in cells transfected with miR-30b and miR-30c, whereas reporter plasmid with mutant sequence of Rab18 produced no change in luciferase activity (Figure 1A,B). Then, we explored whether the
A

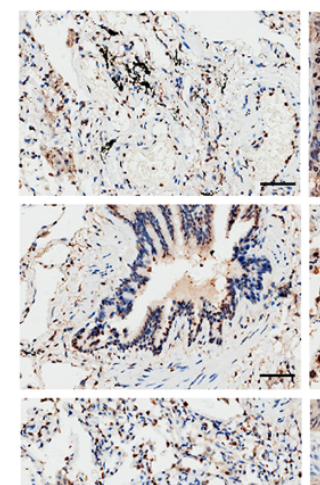

\#3
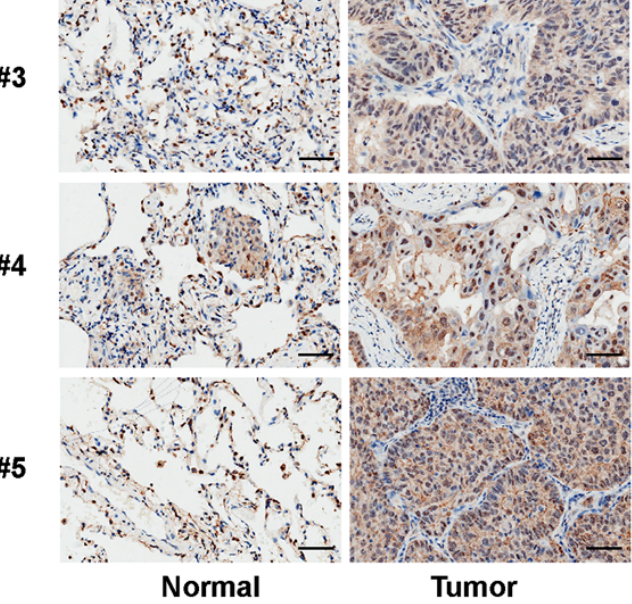

B
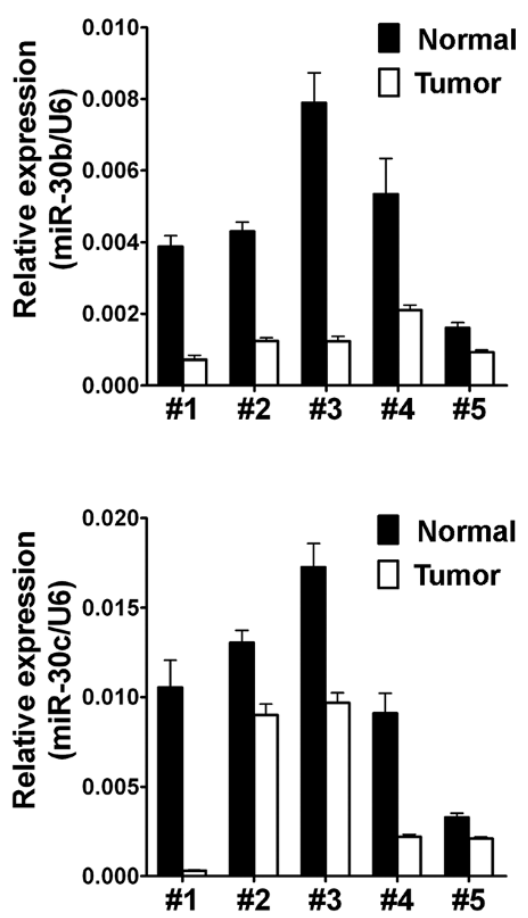

Figure 2 Expression analyses of miR-30b/c and Rab18 in NSCLC tissues. (A) IHC analysis of Rab18 expression in five pairs of lung cancer tissues and their corresponding non-tumor tissues, Brown signal in IHC was considered as positive staining for Rab18. Scale bars, 50 m. (B) qRT-PCR analysis of miR-30b (up) and miR-30c (down) expression in five pairs of lung cancer tissues and their corresponding non-tumor tissues as indicated in (A). The expressions of miR-30b/c were normalized to U6. 
endogenous Rab18 in NSCLC cells was regulated similarly. A549 and H23 cells were transfected with miR-30b or miR-30c, and Rab18 protein levels and mRNA levels were examined by WB and qRT-PCR, respectively. Our results showed that Rab18 mRNA expression was not affected by miR-30b and miR-30c (Figure 1C), while the level of Rab18 protein was consistently and substantially down-regulated by miR-30b and miR-30c (Figure 1D).
Taken together, our results demonstrated that Rab18 was a direct target of miR-30b/c in NSCLC cells.

$\mathrm{miR}-30 \mathrm{~b} / \mathrm{c}$ are low-expressed and Rab18 is high-expressed in NSCLC tissue samples

Prompted by our results that Rab18 was a direct target of miR-30b/c in NSCLC cells, we sought to investigate the association of $\mathrm{miR}-30 \mathrm{~b} / \mathrm{c}$ and Rab18 in NSCLC

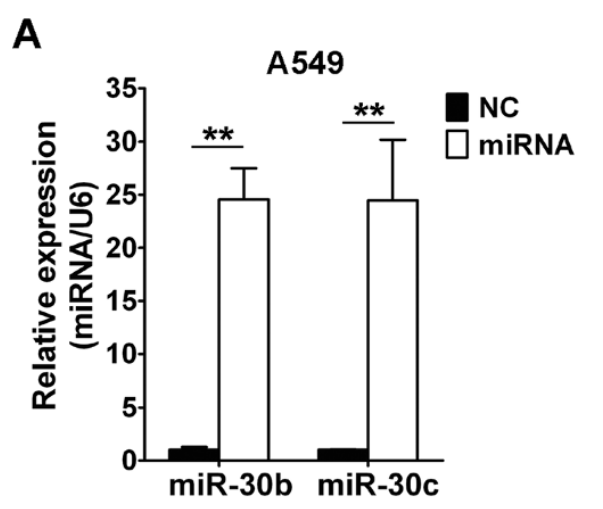

B

C

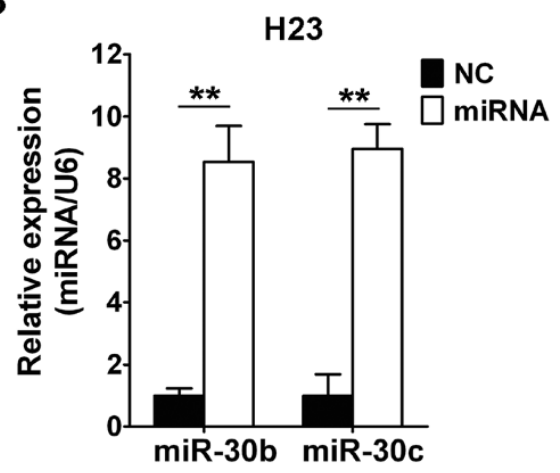

A549
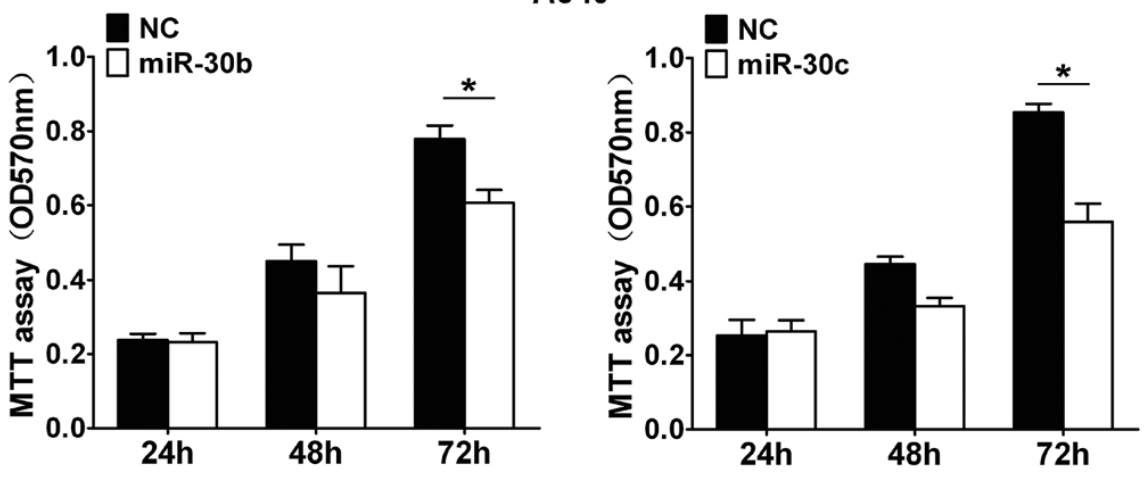

D

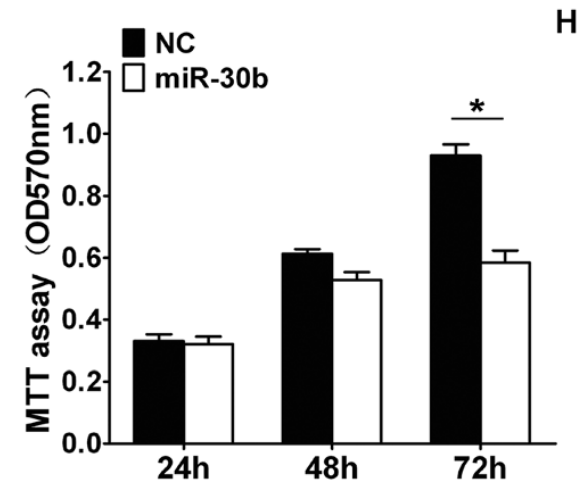

H23

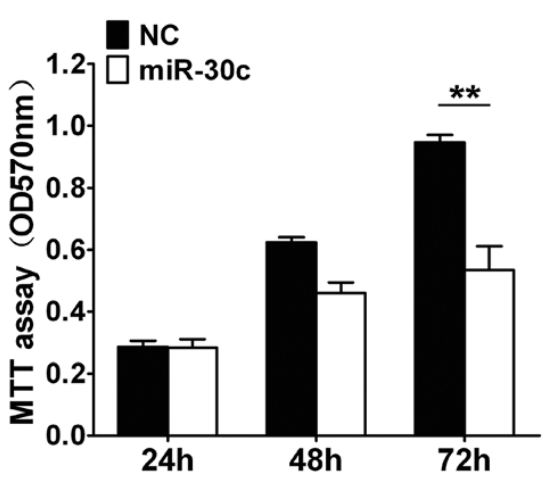

Figure 3 Overexpression of miR-30b/c inhibits NSCLC cells growth in vitro. (A,B) qRT-PCR analysis of miR-30b/c in A549 (A) and H23 (B) cells transfected with miR-30b/c mimics or negative control oligonucleotides. (C,D) The effects of miR-30b/c on A549 (C) and H23 (D) cells proliferation were determined by MTT assay at $24 \mathrm{~h}, 48 \mathrm{~h}$, and $72 \mathrm{~h}$ after transfecting of miR-30b/c mimics or negative control oligonucleotides. $\left({ }^{*} P<0.05,{ }^{* *} P<0.01\right.$, Student's $t$-test). 
tissues. Rab18 protein expression of five pairs of clinical NSCLC and adjacent non-tomor tissues was analyzed by IHC. Our results showed that the protein levels of Rab18 were up-regulated in clinical NSCLC tissues compared with their adjacent non-tomor tissues (Figure 2A). Furthermore, we analyzed the expression of $\mathrm{miR}-30 \mathrm{~b} / \mathrm{c}$ in these five pairs of clinical NSCLC and adjacent non-tumor tissues by qRT-PCR and normalized to an endogenous control (U6 RNA). We found that miR-30b and miR-30c were down-regulated in these five pairs of clinical NSCLC tissues compared with their adjacent non-tomor tissues (Figure 2B). The results suggested that the reduced miR$30 \mathrm{~b} / \mathrm{c}$ expression and increased Rab18 protein expression were frequent events in human NSCLCs tissues.

\section{$\mathrm{miR}-30 \mathrm{~b} / \mathrm{c}$ inhibits NSCLC cell proliferation}

In order to investigate the effect of $\mathrm{miR}-30 \mathrm{~b} / \mathrm{c}$ on NSCLC cell proliferation, miR-30b or miR-30c were transfected into A549 and H23 cells. qRT-PCR results determined that transfection of miR-30b or miR-30c increased their expressions in A549 (Figure 3A) and H23 (Figure 3B) cells. NSCLC cells proliferation was assessed by MTT assay. Our results showed that cellular proliferation gradually declined following transfection with miR-30b or miR-30c in A549 (Figure 3C) and H23 (Figure 3D) cells. Compared with the negative control, treatment of cells with miR-30b or miR-30c led to a decrease in NSCLC cell growth at $72 \mathrm{~h}$, and the inhibitory efficiencies in A549 cells were 25.1\% $(P<0.05)$ and 35.7\% $(P<0.05)$, respectively, and the inhibitory efficiencies in $\mathrm{H} 23$ cells were $37.2 \%(P<0.05)$ and $43.6 \%(P<0.01)$, respectively. Taken together, these results demonstrated that $\mathrm{miR}-30 \mathrm{~b} / \mathrm{c}$ could inhibite the proliferation of NSCLC cells in vitro.

\section{$\mathrm{miR}-30 \mathrm{~b} / \mathrm{c}$ inhibits the proliferation of NSCLC cells via regulation of Rab18}

Since overexpression of $\mathrm{miR}-30 \mathrm{~b} / \mathrm{c}$ suppressed the proliferation of NSCLC cells, and given that Rab18 is a direct target of $\mathrm{miR}-30 \mathrm{~b} / \mathrm{c}$, we hypothesized that the inhibitory effect of $\mathrm{miR}-30 \mathrm{~b} / \mathrm{c}$ on NSCLC cell viability might be achieved via targeting Rab18. In order to investigate this hypothesis, we tested whether RNAi-mediated reduction in Rab18 levels influence the cell growth of NSCLC cells. Treatment of cells with Rab18 siRNA markedly decreased mRNA and protein levels of Rab18 in A549 and H23 cells (Figure 4A and B). MTT assay was performed to determine

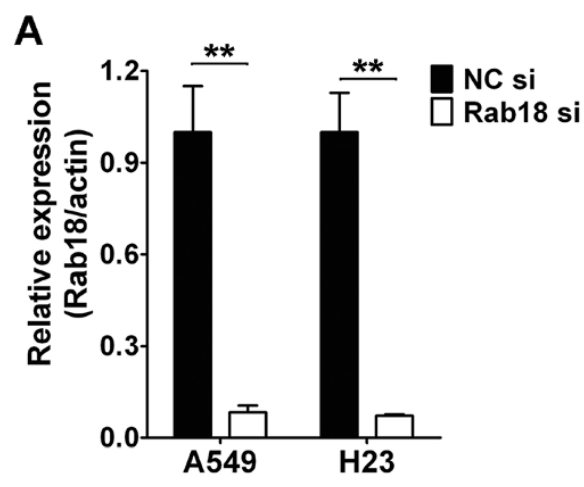

\section{B}

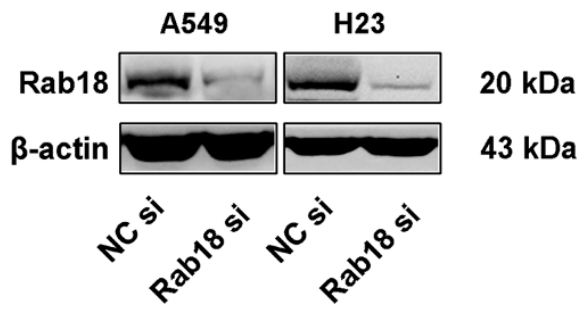

\section{C}
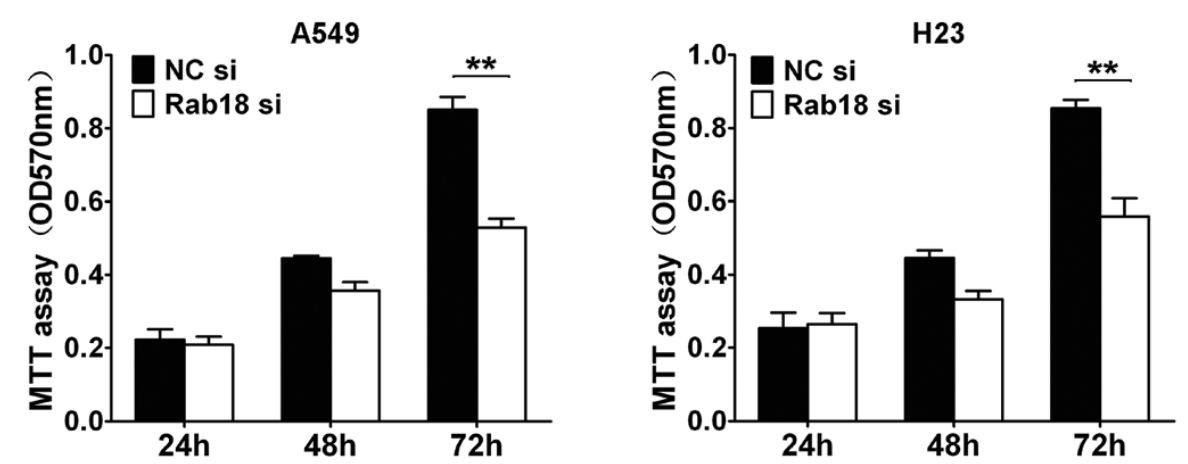

Figure 4 Knockdown of Rab18 inhibits NSCLC cells growth in vitro. (A,B) qRT-PCR (A) and Western blot analysis (B) were performed to determine the expression level of Rab18 after transfection of Rab18 siRNA and control siRNA in A549 and H23 cells. (C, D) The effects of Rab18 siRNA on A549 (C) and H23 (D) cells proliferation were determined by MTT assay at 24 h, 48 h, and $72 \mathrm{~h}$ after transfecting of Rab18 siRNA and control siRNA. $\left({ }^{*} P<0.05,{ }^{* *} P<0.01\right.$, Student's $t$-test). 
the effect of Rab18 siRNA on cell proliferation. The results showed that Rab18 siRNA treatment suppressed cell viability by $34 \%(P<0.01)$ in A549 cells and $36.4 \%(P<0.01)$ in $\mathrm{H} 23$ cells at $72 \mathrm{~h}$, compared with control siRNA (Figure 4C). Our results agreed with the previously study showing that Rab18 was associated with hepatocellular carcinoma (HCC) proliferation. Our results demonstrated that down-regulation of Rab18 expression by $\mathrm{miR}-30 \mathrm{~b} / \mathrm{c}$ contributed, at least in part, to the suppression of the growth of NSCLC cells.

\section{Disscussion}

Recently, attentions have focused on the role of miRNA in tumorigenesis. In this study, we focused on miR-30 which was decreased in several tumor types including NSCLC. Our results showed that Rab18 was one direct functional targets of miR-30b/c in NSCLC cells. Downregulation of miR-30b/c and up-regulation of Rab18 protein levels were also found in NSCLC tissues compared to adjacent non-tumor tissues. Furthermore, ectopic overexpression of miR-30b/c blocked NSCLC cells proliferation in vitro.

The aberrant expression of miRNAs is associated with cancer progression including proliferation, migration, invasion and apoptosis. Deregulation of miRNAs such as miR-221, miR-222, miR-449a, miR-21, miR-205, miR-10b, miR-143 and miR-181a in NSCLC is a key factor underlying tumorigenesis [25]. Human miR-30 is downregulated in several tumor types including NSCLC [20]. This suggests miR-30 is a potential tumor suppressor. These findings prompted us to investigate the regulation of miR-30 in NSCLC cells. Recent studies showed that miR-30a regulated growth of breast cancer cells [26], down-regulation of miR-30 maintained self-renewal and inhibited apoptosis in breast tumor-initiating cells [27], miR-30 regulated B-Myb expression during cellular senescence [28]. However, the role of miR-30 in cancers especially in NSCLC is not very much known. In this study, we confirmed that oncogene Rab18 was directly targeted by $\mathrm{miR}-30 \mathrm{~b} / \mathrm{c}$ in NSCLC cells. Decreased miR-30b/c and increased Rab18 protein expression were also found in NSCLC tissues, which suggested that Rab18 was regulated by $\mathrm{miR}-30 \mathrm{~b} / \mathrm{c}$ in human NSCLC tissues. Human miR-30 family including miR-30a, miR-30b, miR-30c, miR-30d and miR-30e have the samilar sequence. Whether other miR-30 family have the samilar function like miR-30b and miR-30c in NSCLC or other cancer cells remain to be investigated.

Rabs, small G proteins belonging to the Ras superfamily, are regulators of vesicular transport in both exocytic and endocytic pathways in eukaryotic cells [29]. Emerging evidences have revealed the association between dysfunction of the Rab18 and multiple human diseases including cancer [29-31]. It had been reported that Rab18 was involved in the lipogenesis of 3T3-L1 adipocytes [23]. Loss-of-function mutations in Rab18 caused Warburg Micro syndrome [32]. Moreover, Rab18 acted as a novel tumor antigen in medulloblastoma and HCC $[23,33]$. However, the expression of Rab18 is less well known in human cancers especially in NSCLC. In this study, we first reported that Rab18 protein levels were highly expressed in NSCLC tissues compared to matched adjacent nontumor tissues. To investigate the underlying mechanism of up-regulation of Rab18 protein levels in NSCLC. miRNA-binding sites analysis revealed that Rab18 was one direct functional target of miR-30b/c in NSCLC cells. Transfection of miR-30b/c mimics into NSCLC cells led to a significant Rab18 decrease at protein levels but not mRNA levels and inhibition of cellular proliferation. Furthermore, silencing Rab18 expression by siRNA in NSCLC cells also led to inhibition of cellular proliferation. These findings support the hypothesis that decreased expression of Rab18 by miR-30b/c accounts for the suppression of cellular proliferation in NSCLC. Our results agreed with the previous study in HCC which showed that Rab18 was directly targeted and down-regulated at protein levels by miR-429 in HCC cells, but whether the expression of Rab18 was also regulated at transcriptional levels in NSCLC cells just like that by hepatitis B virus X protein stimulation in HCC cells remain to be investigated [23].

\section{Conclusions}

Taken together, we demonstrate that miR-30b/c is downregulated in NSCLC tissues. Overexpression of miR-30b/c directly down-regulates Rab18 and inhibits NSCLC cell proliferation. These data indicate that $\mathrm{miR}-30 \mathrm{~b} / \mathrm{c}$ could serve as a tumor suppressor gene involved in NSCLC pathogenesis.

\section{Abbreviations}

NSCLC: Non-small cell lung cancer; mRNA: Messenger RNA; qRT-PCR: Quantitative reverse transcription-polymerase chain reaction; miRNA: microRNA; FBS: Fetal bovine serum.

\section{Competing interests}

The authors declare that they have no competing interests.

\section{Authors' contributions}

$\mathrm{HL}$ and $\mathrm{BL}$ conceived and designed the experiments. $\mathrm{KZ}$ and $\mathrm{KC}$ performed the experiments. KC collected the samples and analyzed the data. KZ wrote the paper. All authors are in agreement with the content of the manuscript and this submission. All authors read and approved the final manuscript.

\section{Acknowledgments}

This research was made possible with financial support from National Natural Sciences Foundation of China, for the project 81201780.

\section{Author details}

${ }^{1}$ Department of Cardiothoracic Surgery, Changhai Hospital affiliated to Second Military Medical University, Shanghai 200433, China. ${ }^{2}$ Department of Respiratory Medicine, People's Hospital of Tongren City, Guizhou 554300, China. 
Received: 15 January 2014 Accepted: 17 September 2014

Published: 24 September 2014

\section{References}

1. Jemal A, Bray F, Center MM, Ferlay J, Ward E, Forman D: Global cancer statistics. CA Cancer J Clin 2011, 61(2):69-90.

2. Wood AJJ, Spira A, Ettinger DS: Multidisciplinary management of lung cancer. N Engl J Med 2004, 350(4):379-392.

3. Yang L, Parkin DM, Ferlay J, Li L, Chen Y: Estimates of cancer incidence in China for 2000 and projections for 2005. Cancer Epidemiol Biomark Prev 2005, 14(1):243-250.

4. Ferlay J, Shin HR, Bray F, Forman D, Mathers C, Parkin DM: Estimates of worldwide burden of cancer in 2008: GLOBOCAN 2008. Int J Cancer 2010, 127(12):2893-2917.

5. Stefani G, Slack FJ: Small non-coding RNAs in animal development. Nat Rev Mol Cell Biol 2008, 9(3):219-230.

6. Fabian MR, Sonenberg N, Filipowicz W: Regulation of mRNA translation and stability by microRNAs. Annu Rev Biochem 2010, 79:351-379.

7. Zhao J-J, Yang J, Lin J, Yao N, Zhu Y, Zheng J, Xu J, Cheng JQ, Lin J-Y, Ma X: Identification of miRNAs associated with tumorigenesis of retinoblastoma by miRNA microarray analysis. Childs Nerv Syst 2009, 25(1):13-20.

8. Hassan O, Ahmad A, Sethi S, Sarkar FH: Recent updates on the role of microRNAs in prostate cancer. J Hematol Oncol 2012, 5(9):13.

9. Du L, Pertsemlidis A: microRNA regulation of cell viability and drug sensitivity in lung cancer. Expert Opin Biol Ther 2012, 12(9):1221-1239.

10. Visone R, Croce CM: MiRNAs and cancer. Am J Pathol 2009, 174(4):1131-1138.

11. Acunzo M, Visone R, Romano G, Veronese A, Lovat F, Palmieri D, Bottoni A, Garofalo M, Gasparini P, Condorelli G: miR-130a targets MET and induces TRAIL-sensitivity in NSCLC by downregulating miR-221 and 222. Oncogene 2011, 31(5):634-642.

12. Jeon HS, Lee SY, Lee EJ, Yun SC, Cha EJ, Choi E, Na MJ, Park JY, Kang J, Son JW: Combining microRNA-449a/b with a HDAC inhibitor has a synergistic effect on growth arrest in lung cancer. Lung Cancer 2012, 76(2):171-176.

13. Zhang J-G, Wang J-J, Zhao F, Liu Q, Jiang K, Yang G-H: MicroRNA-21 (miR-21) represses tumor suppressor PTEN and promotes growth and invasion in non-small cell lung cancer (NSCLC). Clin Chim Acta 2010, 411(11):846-852.

14. Lebanony D, Benjamin H, Gilad S, Ezagouri M, Dov A, Ashkenazi K, Gefen N, Izraeli S, Rechavi G, Pass H: Diagnostic assay based on hsa-miR-205 expression distinguishes squamous from nonsquamous non-small-cell lung carcinoma. J Clin Oncol 2009, 27(12):2030-2037.

15. Liu X, Sempere LF, Guo Y, Korc M, Kauppinen S, Freemantle SJ, Dmitrovsky $\mathrm{E}$ : Involvement of microRNAs in lung cancer biology and therapy. Trans/ Res 2011, 157(4):200-208.

16. Gao W, Yu Y, Cao H, Shen H, Li X, Pan S, Shu Y: Deregulated expression of miR-21, miR-143 and miR-181a in non small cell lung cancer is related to clinicopathologic characteristics or patient prognosis. Biomed Pharmacother 2010, 64(6):399-408.

17. Yu F, Deng $H$, Yao H, Liu Q, Su F, Song E: Mir-30 reduction maintains self-renewal and inhibits apoptosis in breast tumor-initiating cells. Oncogene 2010, 29(29):4194-4204.

18. Feber A, Wilson GA, Zhang L, Presneau N, Idowu B, Down TA, Rakyan VK, Noon LA, Lloyd AC, Stupka E: Comparative methylome analysis of benign and malignant peripheral nerve sheath tumors. Genome Res 2011, 21(4):515-524

19. Quintavalle C, Donnarumma E, laboni M, Roscigno G, Garofalo M, Romano G, Fiore D, De Marinis P, Croce CM, Condorelli G: Effect of miR-21 and miR-30b/c on TRAlL-induced apoptosis in glioma cells. Oncogene 2012, 31:4001-4008

20. Guan P, Yin Z, Li X, Wu W, Zhou B: Meta-analysis of human lung cancer microRNA expression profiling studies comparing cancer tissues with normal tissues. J Exp Clin Cancer Res 2012, 31(1):54.

21. Chen C, Ridzon DA, Broomer AJ, Zhou Z, Lee DH, Nguyen JT, Barbisin M, Xu NL, Mahuvakar VR, Andersen MR: Real-time quantification of microRNAs by stem-loop RT-PCR. Nucleic Acids Res 2005, 33(20):179.

22. Li J, Kong X, Zhang J, Luo Q, Li X, Fang L: MiRNA-26b inhibits proliferation by targeting PTGS2 in breast cancer. Cancer Cell Int 2013, 13(1):7.

23. You $X$, Liu F, Zhang T, Li Y, Ye L, Zhang X: Hepatitis B virus $X$ protein upregulates oncogene Rab18 to result in the dysregulation of lipogenesis and proliferation of hepatoma cells. Carcinogenesis 2013 34(7):1644-1652.
24. Helwak A, Kudla G, Dudnakova T, Tollervey D: Mapping the human miRNA interactome by CLASH reveals frequent noncanonical binding. Cell 2013, 153(3):654-665.

25. Liang $Y$ : An expression meta-analysis of predicted microRNA targets identifies a diagnostic signature for lung cancer. BMC Med Genet 2008, 1(1):61.

26. Ouzounova M, Vuong T, Ancey P-B, Ferrand M, Durand G, Kelm FL-C, Croce C, Matar C, Herceg Z, Hernandez-Vargas H: MicroRNA miR-30 family regulates non-attachment growth of breast cancer cells. BMC Genomics 2013, 14(1):139.

27. Liu C, Tang DG: MicroRNA regulation of cancer stem cells. Cancer Res 2011, 71(18):5950-5954.

28. Martinez I, Cazalla D, Almstead LL, Steitz JA, DiMaio D: miR-29 and miR-30 regulate B-Myb expression during cellular senescence. Proc Natl Acad Sci 2011, 108(2):522-527.

29. Chia WJ, Tang BL: Emerging roles for Rab family GTPases in human cancer. Biochim Biophys Acta 2009, 1795(2):110-116.

30. Tang BL, Ng EL: Rabs and cancer cell motility. Cell Motil Cytoskeleton 2009, 66(7):365-370.

31. Cheng KW, Lahad JP, Gray JW, Mills GB: Emerging role of RAB GTPases in cancer and human disease. Cancer Res 2005, 65(7):2516-2519.

32. Bem D, Yoshimura S-I, Nunes-Bastos R, Bond FF, Kurian MA, Rahman F, Handley MTW, Hadzhiev Y, Masood I, Straatman-Iwanowska AA: Loss-offunction mutations in RAB18 cause Warburg micro syndrome. Am J Hum Genet 2011, 88(4):499-507.

33. Behrends U, Schneider I, Rössler S, Frauenknecht H, Golbeck A, Lechner B, Eigenstetter G, Zobywalski C, Müller-Weihrich S, Graubner U: Novel tumor antigens identified by autologous antibody screening of childhood medulloblastoma cDNA libraries. Int J Cancer 2003, 106(2):244-251.

doi:10.1186/1471-2407-14-703

Cite this article as: Zhong et al.: microRNA-30b/c inhibits non-small cell lung cancer cell proliferation by targeting Rab18. BMC Cancer 2014 14:703.

\section{Submit your next manuscript to BioMed Central and take full advantage of:}

- Convenient online submission

- Thorough peer review

- No space constraints or color figure charges

- Immediate publication on acceptance

- Inclusion in PubMed, CAS, Scopus and Google Scholar

- Research which is freely available for redistribution 